\title{
Study of Gas-Water Flow Inside of a Horizontal Passive Cyclonic Gas-Liquid Phase Separator System Using Displacement-Current Phase Tomography
}

\author{
Joshua N. Sines ${ }^{1}$, Benjamin J. Straiton ${ }^{1}$, Christopher E. Zuccarelli ${ }^{1}$, Qussai M. Marashdeh ${ }^{1}$, Fernando L. \\ Teixeira ${ }^{2}$, Liang-Shih Fan ${ }^{2}$, and Brian J. Motil ${ }^{3}$
}

${ }^{I}$ Tech4Imaging LLC, Columbus, OH; ${ }^{2}$ The Ohio State University, Columbus, OH; ${ }^{3} N A S A$ Glenn Research Center, Cleveland, $\mathrm{OH}$

\begin{abstract}
Passive cyclonic gas-liquid separators (PCGLSs) are commonly used in microgravity conditions where gravity settling separation is difficult or impossible. In this study, displacement-current phase tomography (DCPT) is used to measure various features of the gasliquid flow inside of a PCGLS. The liquid holdup, liquid angular velocity, and gas core size are investigated. The liquid holdup is also measured in a gas-liquid flow that simulates the injection flow for a PCGLS. It is found that the gas core contracts and expands in a periodic motion as air is injected with water. This motion becomes more noticeable as the air flow rate is increased. It is also found that the liquid layer angular velocity has a positive linear trend with the air flow rate
\end{abstract}

Key words: Displacement-Current Phase Tomography; Electrical Capacitance Phase Tomography; Capacitance Tomography; Cyclonic Separation; Liquid Cyclone; Gas Core; Gas-Liquid Separator; Two-phase Flow; Liquid Holdup; Flow Imaging; Liquid Velocity; Gasliquid Mixture; Gas Core Size

Correspondence to: Joshua N. Sines

Tech4Imaging LLC

1910 Crown Park Ct.

Columbus, OH 43235

Telephone: 614-725-1778

E-mail: josh@tech4imaging.com under constant water flow rates. A basic linear relation is derived to relate the liquid angular velocity to the air and water flow rates. All DCPT and electrical capacitance phase tomography (ECVT) results closely match the visual confirmation methods used for each flow feature.

\section{INTRODUCTION}

Many multiphase flows require a form of gasliquid separation. The most common method for gas-liquid separation is to use gravity to settle out the fluids. However, this method of separation is not practical or may be impossible under special circumstances. One of these situations is in the microgravity environment encountered in space. Because there is little to no gravity in these environments, the fluids do not naturally separate when left to settle. For these applications, a passive cyclonic gas-liquid separator (PCGLS) is preferred because of its low weight and size, low power consumption, and higher separation efficiency when compared with other passive inertia devices (Wang et al., 2016). A PCGLS consists of a cylindrical tube with a tangentially mounted gas-liquid injection nozzle. One end of the PCGLS is used as a gas outlet and the other is used as the liquid outlet. The PCGLS separates the gas and liquid by utilizing the centrifugal forces created by the swirling of the gas and liquid around the separator. The denser liquid is pushed outwards creating a gas core in the center of the 


\section{Sines et al. -- Study of Flow in a Phase Separator Using DCPT}

separator. An axial swirl-stop baffle plate is inserted in front of the liquid outlet to stop the rotation of the two phases and separate the water from the gas. The pressure inside the system must be balanced with the rate of gas-liquid injection to achieve complete separation. If the system is unbalanced then some liquid may exit from the gas outlet and some gas may exit from the liquid outlet.

Many studies have been conducted on PCGLS devices both on the ground and in microgravity conditions. These studies have used techniques such as acoustic gauge and electrical resistance tomography to investigate the effect of separator design and operation on the gas core and velocity profile (Barbu et al., 2006; Hoyt et al., 2011; Hoyt et al., 2013; Kang et al., 2014). Electrical capacitance volume tomography (ECVT) has also been used to study the flow pattern, gas core location, and angular velocity of a PCGLS (Wang et al., 2016).

The present study expands upon the ECVT work previously conducted by testing a variety of air and water flow rates, utilizing a high speed camera and tachometer to confirm measurements, and by using displacement-current phase tomography (DCPT) (Gunes et al., 2017) to derive gas core images, liquid holdup, and angular velocity of the liquid layer inside the separator. DCPT is also used in this study to measure the liquid holdup in an air-water flow that simulates the type of flow injected into the PCGLS. DCPT, is an extension of ECVT that can be used to better examine conductive flows such as the PCGLS (Gunes et al., 2017). A high speed camera is used to measure the size of the air core and a tachometer is used to measure the angular velocity of the liquid layer. DCPT is used to generate reconstructed images of the gas core and liquid layer inside of the separator. These images are then used to determine the size of the gas core and inversely the liquid holdup. The real-time liquid holdup is then measured using DCPT and observed to closely match the estimated liquid holdup from the high speed camera images. The angular velocity of the liquid layer is also measured using DCPT data and is found to be proportional to the gas flow rate at a constant water flow rate. Finally, the liquid holdup in a vertical two-phase flow is measured both visually and with an ECVT system.

\section{Electrical Capacitance Phase Tomography Theory}

ECVT

Electrical capacitance volume tomography (ECVT) is a non-invasive tomography technique developed to measure multiphase flows (Warsito et al., 2007). Flexible capacitance plates distributed along the outside wall of the measurement region are used with an ECVT data acquisition system (DAS) to collect real-time measurements of inter-electrode capacitances. These capacitance measurements can then be used to derive information of the measured flow. An optimization reconstruction algorithm can also be employed to generate 3-D images of the sensing domain. ECVT has been used successfully in gasliquid systems including a previous study on a PCGLS (Wang et al., 2016).

\section{$D C P T$}

ECVT measurements are collected using alternating current (AC) signals. As such, the measured current has two components - the quadrature component (used to extract the capacitance) and the in-phase component. Typical ECVT measurements and images only utilize the quadrature component as the magnitude of the phase component is relatively small. However, this phase component contains valuable information as it depends on the conductivity (dielectric loss) present in the measurement region. Displacement-current phase tomography (DCPT) is developed to utilize this phase component to derive information and images of flows. DCPT is especially useful for lossy environments, such as a water continuous system, because of the relationship between phase and the systems dielectric loss. DCPT has been shown in previous studies to be better for imaging air-water systems than ECVT (Gunes et al., 2017). DCPT uses the same data collection system as ECVT and can be collected at the same time as capacitance measurements. This allows for both measurement methods to be utilized if needed (Gunes et al., 2017). In general, DCPT is best suited for systems containing at least one high-conductivity medium, like water, whereas ECVT is best suited for systems containing low-conductivity media, such as an oil-air flow. 


\section{MATERIALS AND METHODS}

\section{Experimental Setup}

PCGLS setup

The PCGLS is made out of a clear acrylic tube with an outer diameter (OD) of 3.5 in (8.89 $\mathrm{cm})$ and an inner diameter (ID) of 3 in $(7.62 \mathrm{~cm})$. A 0.25 in $(0.635 \mathrm{~cm})$ ID injection nozzle is attached to the outer wall near the gas outlet. A swirl stopping baffle plate is placed 2 in $(5.08 \mathrm{~cm})$ from the liquid outlet end of the cylinder. The baffle plate is used to stop the vortex flow structure before it reaches the liquid outlet. The PCGLS cylinder length is about 9 in $(22.86 \mathrm{~cm})$, meaning the total vortex region length, between the gas outlet and baffle plate, is about 7 in (17.78 $\mathrm{cm})$ long. The air outlet has an OD of 0.375 in $(0.95 \mathrm{~cm})$ and an ID of 0.125 in $(0.32 \mathrm{~cm})$. The water outlet has an ID of 0.875 in $(2.22 \mathrm{~cm})$. A basic diagram of the PCGLS can be seen in Figure 1.

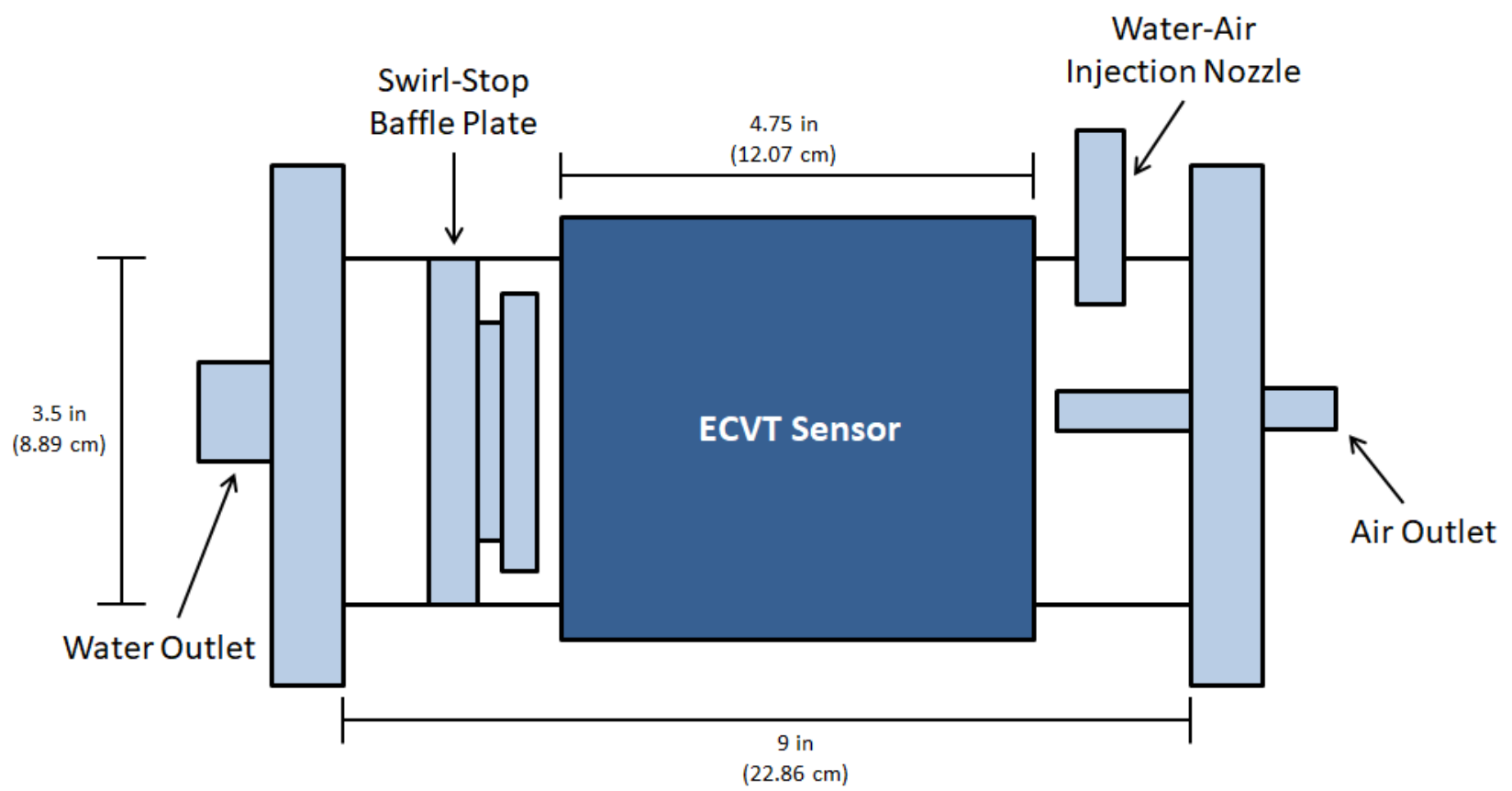

Figure 1. Diagram of test passive cyclonic gas-liquid separators with an electrical capacitance phase tomography (ECVT) sensor placed around it. The ECVT sensor is placed such that almost the entire gas core is within the sensing region.

An ECVT sensor with an inner diameter of 3.5 in $(8.89 \mathrm{~cm})$ and a length of 4.75 in $(12.07$ $\mathrm{cm})$ is placed around the vortex region of the PCGLS. This sensor has 36 plates arranged in 3 layers, giving it 12 plates per layer. ECVT sensors have improved spatial resolution as the number of sensor plates are increased (Wang et al., 2015). The chosen sensor design (3 layers x 12 plates per layer) provides the best balance of axial and radial imaging resolution for this experiment. The data capture rate is 80 frames per second. Figure 2 is a photograph of the sensor placed around the described PCGLS.

The PCGLS is installed horizontally on a flow loop support frame. A water pump is used to circulate the water through the loop and PCGLS with a turbine flow meter placed inline after the pump to measure the water volumetric flow rate. A ball valve is used to control the water flow rate. The air is supplied by an air compressor with its volumetric flow rate measured and controlled using a rotameter.

The water and air are combined using a Yconnector and allowed a 22 in $(55.88 \mathrm{~cm})$ section of 0.75 in $(1.905 \mathrm{~cm})$ ID flow tubing to $\mathrm{mix}$ before being injected into the separator. After separation the water is recycled back into the system and the air is directed into the atmosphere. A ball valve is installed in the water outlet tubing to adjust the back pressure, thus controlling the 


\section{Sines et al. -- Study of Flow in a Phase Separator Using DCPT}

gas core pressure and size inside the separator. The water outlet valve is adjusted to the point where all air is purged from the separator during water only injection and held constant through all experiments. Figure 3 shows a diagram of the PCGLS test flow loop.

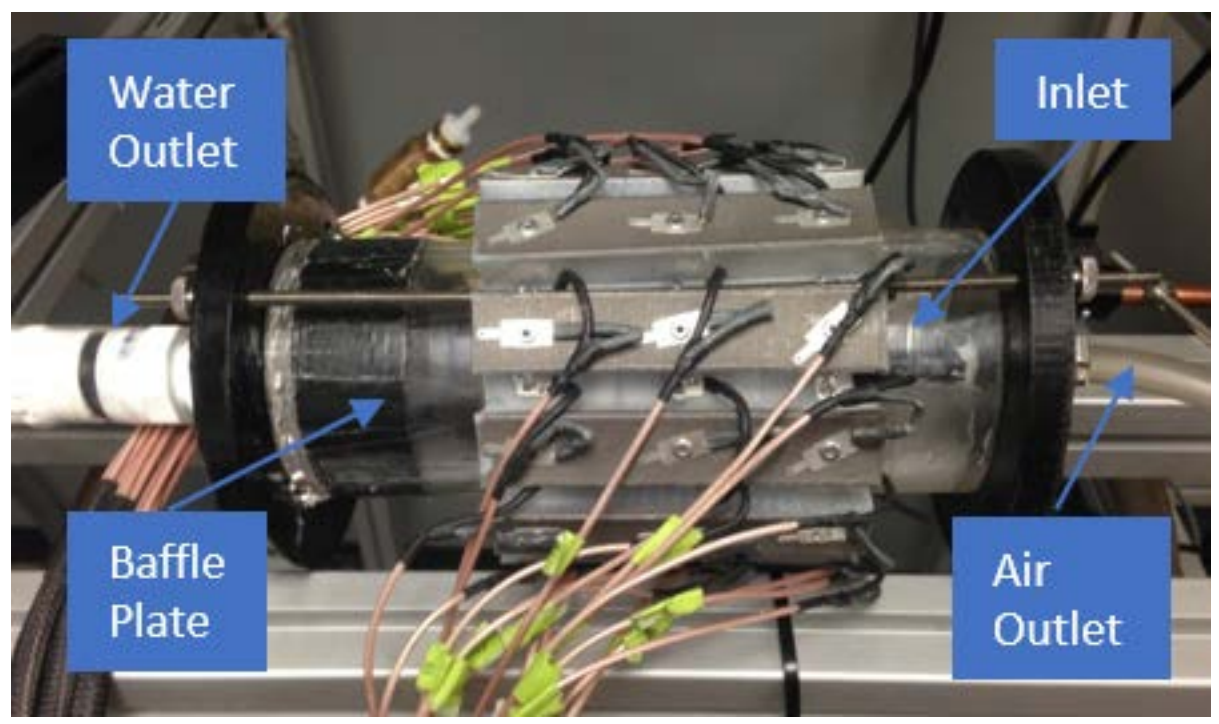

Figure 2. Photograph of the passive cyclonic gas-liquid separators with the electrical capacitance phase tomography sensor placed around it. The cables coming out of the sensor connect each capacitor plate to the data acquisition system.

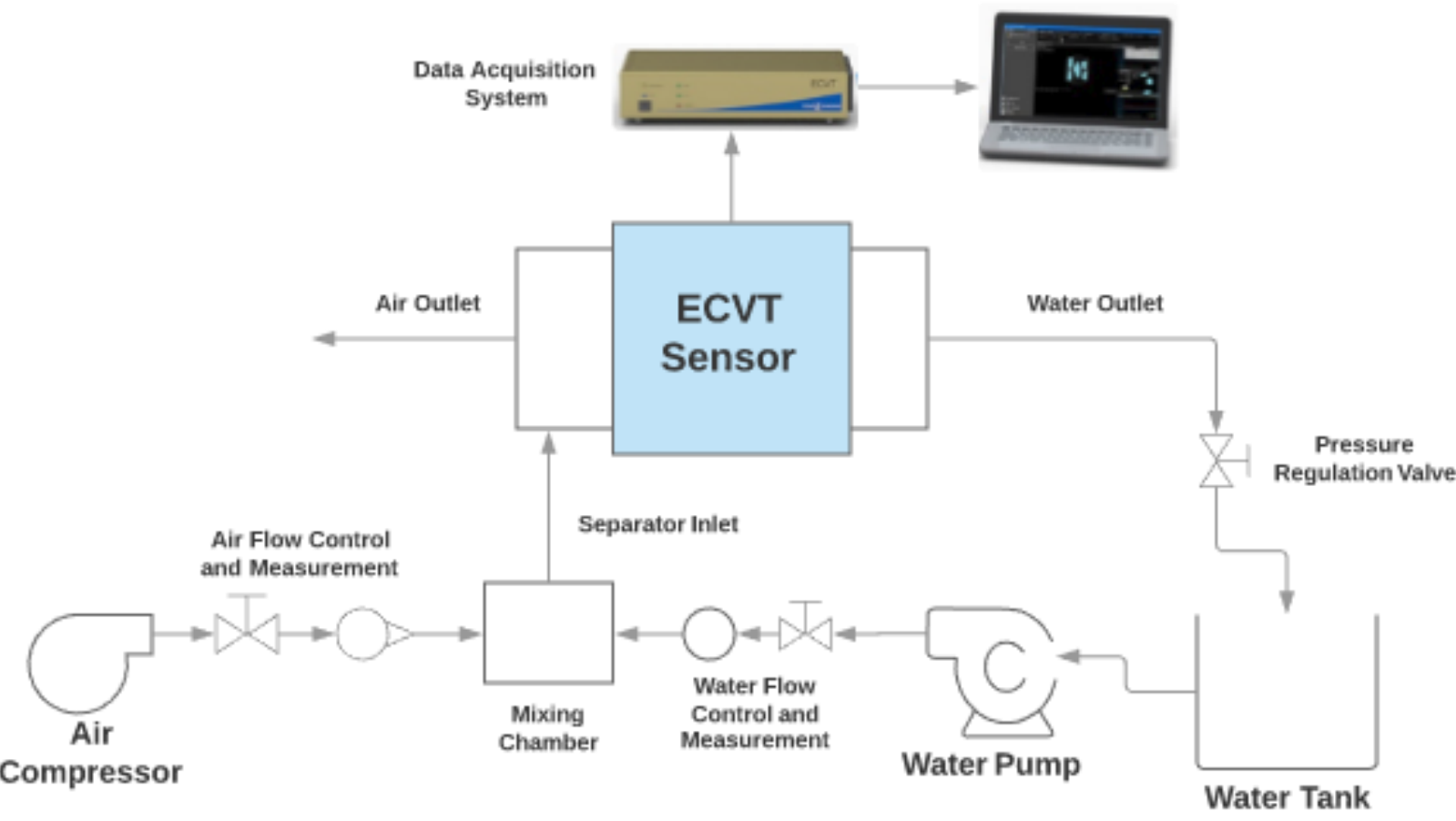

Figure 3. Passive cyclonic gas-liquid separators (PCGLS) flow loop diagram. An air compressor supplies the air flow and a water pump supplies the water flow. The two phases are mixed in a mixing chamber before being injected into the PCGLS. 
The ECVT sensor is calibrated on the PCGLS before the experiments are conducted. For this process ECVT data is collected at an empty state and a full state. The empty state data corresponds to the sensing region being filled with the lowest permittivity material in the system, air in this case. The full state data corresponds to the sensing region being filled with the highest permittivity material, water in this case. This process calibrates settings within the DAS for optimal operation based on the flow materials. This also normalizes the data from the ECVT system between 0 and 1, corresponding to the empty and full states, respectively.

The PCGLS used in this experiment is relatively small compared to potential space applications of this system. The flow behavior of the liquid in the PCGLS is expected to vary as the section size is increased. At smaller section IDs, such as in this experiment, the wall friction has a higher impact on the velocity profile and phase distribution. However, the ECVT sensing technique is scale invariant as it operates in the quasi-static range of the spectrum. In this range, the excitation signal wavelength is orders of magnitude larger than the PCGLS size. For larger sizes, this measurement technique can still be used to study the impact of PCGLS size on the fluid dynamics in different size ranges.

All experiments are conducted at standard temperature and pressure. The water temperature is $20^{\circ} \mathrm{C}$.

\section{Air-water inlet flow setup}

The air-water inlet liquid holdup experiments are conducted on a vertical flow loop at the NASA Glenn Research Center. The flow loop consisted of an up flow section and a down flow section with quick close valves (QCVs) in both sections to stop the flow for volume fraction measurements. A separate flow loop is used for this testing to achieve a better variety of liquid holdup and flow regimes and to better verify measurements using the QCVs. The loop is placed vertically to limit the effects of gravity on the flow and better simulate flow regimes seen in a zero gravity environment. Flow regimes observed in the vertical sections consisted of bubble, churn/transitional, annular, and slug flows which match the four main flow regimes observed in microgravity (Lowe and Rezkallah, 1999).
The flow loop test section is made out of polycarbonate tubing with a 1 in $(2.54 \mathrm{~cm})$ OD and 0.75 in $(1.905 \mathrm{~cm})$ ID. A 12 plate, 2 layer (6 plates per layer) ECVT sensor is placed around both the up flow and down flow test sections. For this test setup a 1 layer sensor could be used to adequately measure liquid holdup. However, 2 layers are used to provide additional data channels to make up for any faulty data points that may arise. The ECVT sensor is calibrated with air and water as the empty and full states, respectively, before experiments are conducted. The data capture rate is 125 frames per second. This sensor has a higher frame rate than the PCGLS because it has less plates. This means that the DAS has less plate pairs to activate and measure, increasing the speed at which the system collects data. Figure 4 shows a basic diagram of the test section used. Water flow rates ranging from 3.79 LPM to 18.95 LPM and air flow rates ranging from 1 SLPM to 50 SLPM are used during this experiment.

\section{Non-ECVT Flow Feature Measurement}

\section{PCGLS}

Under liquid only injection conditions air is purged from the system by the pressure placed on the liquid outlet. Thus, the liquid only injection flows consist solely of a rotating water layer with no air. Under all gas flow test conditions, a gas core forms in the center of the separator with a liquid layer forming along the edge of the separator. It is observed that, when an air-water mixture is injected, air bubbles appear in the water layer in the region between the mixture inlet and gas outlet. Beyond this region the bubbles combine with the gas core, creating distinct layers of gas and liquid. Figure 5 shows three photographs of the air and water layers under a constant water flow rate and three different air flow rates. It was observed that the air core may be off-centric, especially at lower air flow rates. Under the steady state conditions tested in this experiment, the liquid layer can be treated as a rigid body as it rotates around the gas core (Ellis, 2006). It is also observed that the gas core has a pulse like movement where it continuously expands and contracts. This movement is very small but becomes more noticeable as the air flow rate increases. It is possible that this pulse like movement is caused by the wave-like structure at 
the interface between the air core and water layer. This wave-like structure can be seen along the edges of the air cores in Figure 5. It is these irregularities that are utilized to calculate the rotational velocity of the liquid layer as outlined in the PCGLS Angular Velocity section below.

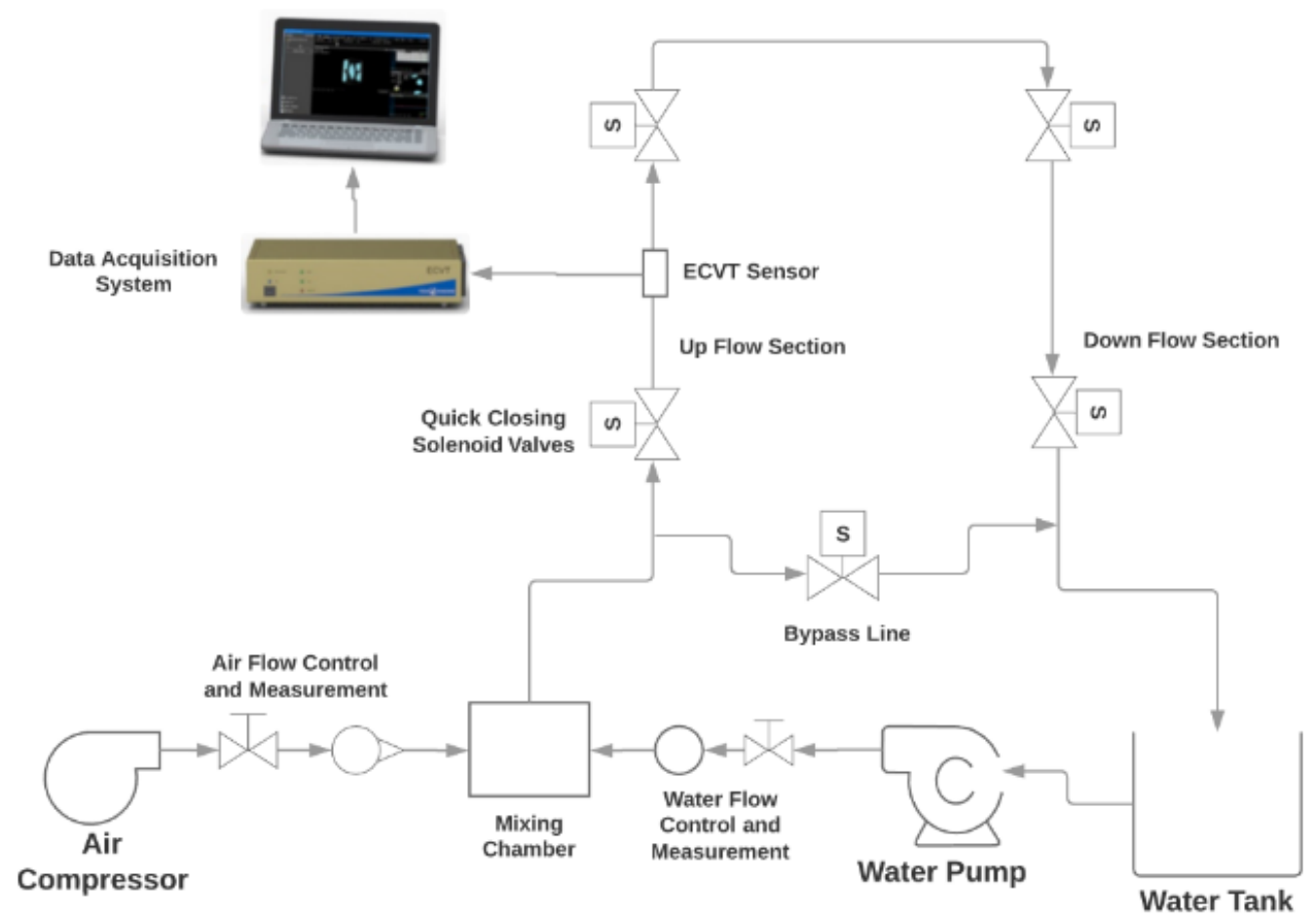

Figure 4. Air-water test flow loop diagram. The test section is oriented vertically to negate the effects of gravity on the flow. Quick close valves are used to stop the flow and measure the amount of water that settled, allowing for the calculation of the average liquid holdup.

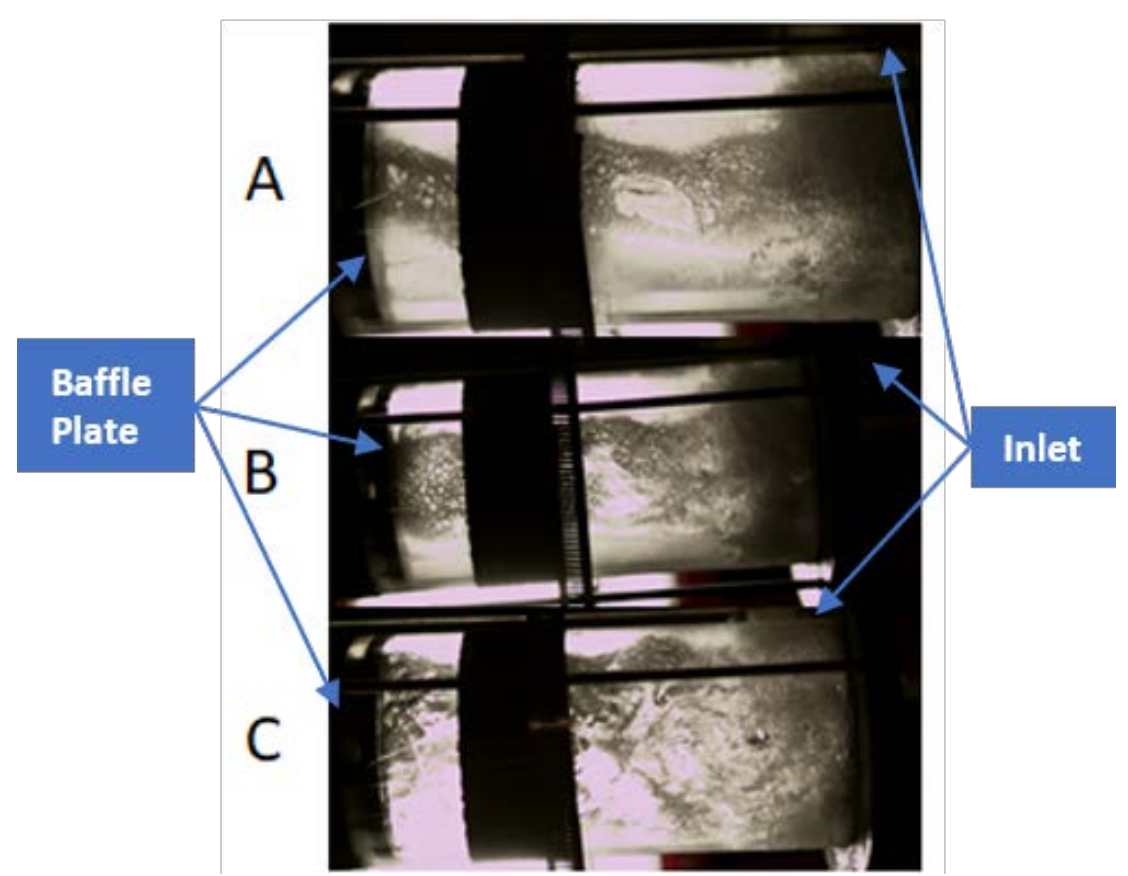

Figure 5. Photographs of three air cores corresponding to a water flow rate of $18.95 \mathrm{LPM}$ and air flow rates of (A) 4.72 SLPM, (B) 9.44 SLPM, and (C) 14.15 SLPM. 


\section{Sines et al. -- Study of Flow in a Phase Separator Using DCPT}

In order to determine the ECVT system accuracy, baseline separator features are measured without the ECVT sensor on the separator. The liquid holdup inside the separator is measured using a high speed video camera. The videos are analyzed to measure the radius of the gas core inside the separator. Distortion due to the water and acrylic tube are accounted for by comparing the size of the core to the size of the baffle plate using equation 1 where $r_{c}$ is the air core radius, $r_{b}$ is the baffle plate radius, and $r_{c i}$ and $r_{b i}$ are the measured radii of the core and baffle plate in the image.

$$
r_{c}=r_{b} * \frac{r_{c i}}{r_{b i}}
$$

Since the actual size of the baffle plate is known, the actual size of the core can be calculated easily by using the ratio of the size of the air core and baffle plate in the image. This measurement method assumes that the baffle plate is distorted equally to the air core. The measured gas core sizes are plotted against the air flow rate at different water flow rates, as can be seen in Figure 6.

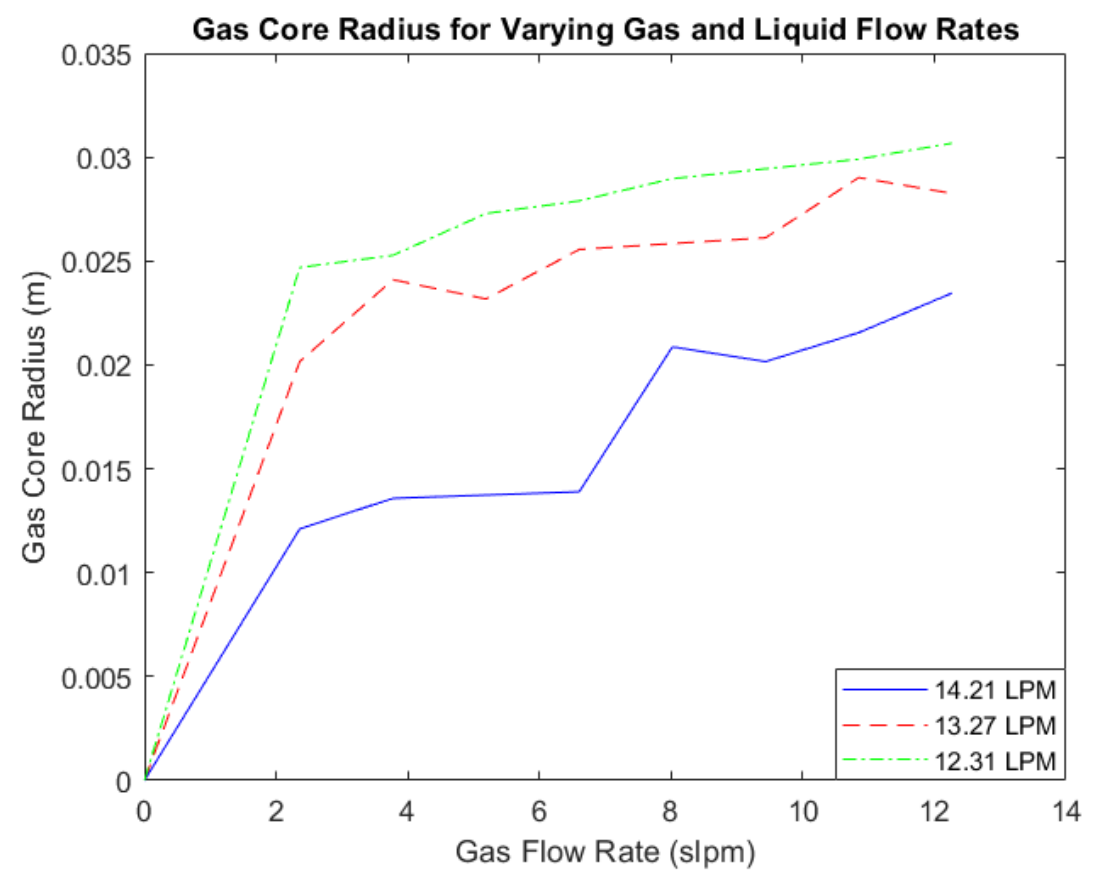

Figure 6. Gas core radius at increasing air flow rates and three constant water flow rates.

Previous research has shown that the liquid holdup inside of the separator due to the gas injection rate follows a similar curve as the ones in Figure 6 (Hoyt, 2013).

To measure the rotational velocity of the water in the separator, a tachometer is used in conjunction with a cylindrical ABS (Acrylonitrile Butadiene Styrene) plastic rod, covered in reflective tape, placed inside the separator. Three sets of rotational velocity measurements with air injection are collected and averaged. The measurements are then plotted against the air flow rate, as can be seen in Figure 7.
As Figure 7 shows, the rotational velocity decreases initially when air is introduced (from 0 slpm gas flow rate to $2.3 \mathrm{slpm}$ ), then increases in a generally linear shape as the air flow rate is increased. Figure 8 shows the rotational velocity measurements plotted against water flow rate under liquid-only conditions. This graph suggests that the angular velocity is proportional to liquid flow rate under liquid-only injections, as is observed in previous research on similar separators (Wang et al., 2016; Ellis, 2006). A linear fit can be applied to derive the relationship between the water injection rate and the angular 


\section{Sines et al. -- Study of Flow in a Phase Separator Using DCPT}

velocity inside the PCGLS. The linear relation for this study can then be expressed as

$$
\Omega(R P M)=59.26 W(L P M)-183
$$

where $\Omega$ is the angular velocity of the water layer in rotations per minute and $\mathrm{W}$ is the water injection rate in liters per minute. As low water flow rates were not tested in this experiment it cannot be assumed that the linear fit would still apply under these conditions.

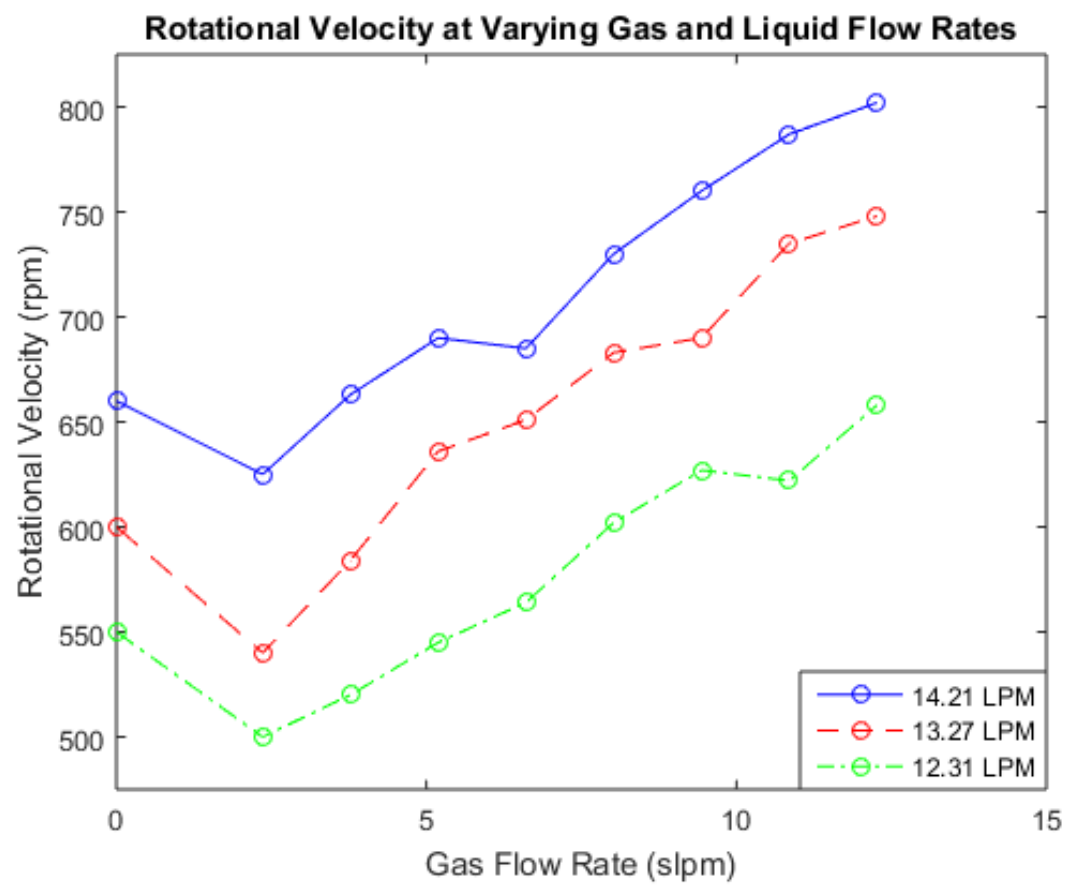

Figure 7. Rotational velocity at increasing air flow rates and three constant water flow rates.

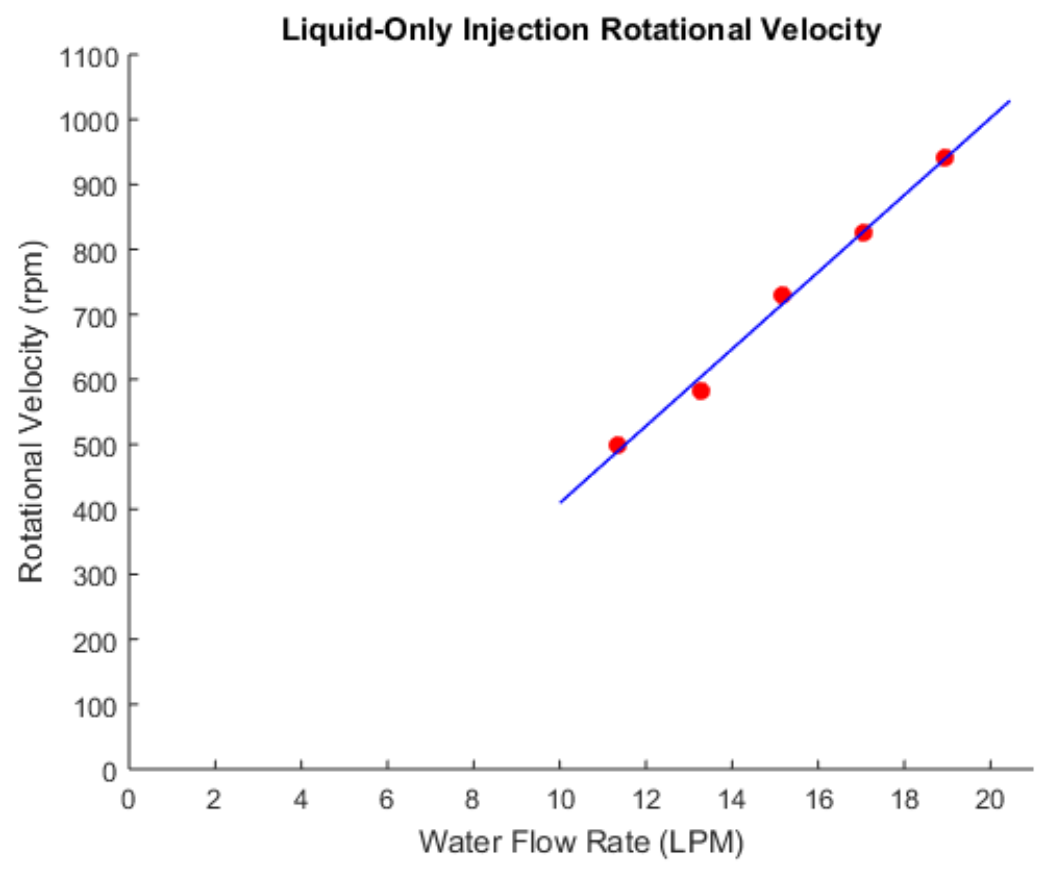

Figure 8. Rotational velocity of liquid-only injections at increasing water flow rates. 
The use of a tachometer to measure the rotational velocity of the water layer serves two purposes in this study. The first purpose is to provide rotational velocity data from a secondary measurement technique to compare to the ECVT results. Second, the tachometer can be used to understand how the rotational velocity relates to the water flow rate under liquid-only injection conditions. The ECVT system utilizes irregularities in the interface between the gas and liquid layers to measure rotational velocity. In this experiment, there is no gas core under liquid-only injection conditions meaning that the sensor has no interface to measure the rotational velocity. The liquid-only injection curve developed from tachometer data is used in Equation 6 to quantify how the air and water injection flow rates affect the rotational velocity of the water layer.

Air-water inlet flow

The liquid holdup inside the test sections are measured visually using the QCVs. When the flow appears to reach a stable flow condition, data is collected with the ECVT system for 30 seconds. The QCVs are then closed and the water allowed to settle at the bottom of the test section. The liquid holdup is then calculated by measuring the height of the water and dividing by the total height of the test section. The QCV measurement process is conducted three times for each water and air inlet condition to get an average liquid holdup value.

The flow regimes observed in the up flow section consist of a bubble flow at low air flow rates that slowly become a pulsating churn flow at higher air flow rates. The down flow section also has a bubble flow at low air flow rates but this section quickly transforms into an annular/falling film flow as the air flow rate is increased. As such, a small change in the air flow rate is observed to cause a larger change in the down flow section than in the up flow section. This allows for lower liquid holdup values to be tested in the down flow section when the water flow rate is decreased and the air flow rate is increased.

\section{RESULTS}

\section{DCPT Flow Measurement and Analysis}

PCGLS gas core imaging

Figure 9 shows a typical real-time DCPT reconstructed image for the liquid and gas distribution inside the separator. Several cross sectional slices perpendicular to the cylinder axis are shown. The reconstructed 2-D images are used to show the shape of the gas core and the liquid distribution within the PCGLS. In the figure, the gas core can clearly be seen as the black and red region. The water is represented by the light blue and white region, with the darker blue pixels representing a region with an air-water interface. These 2-D images can be used to analyze the change in gas core size at various inlet conditions. In Figure 9 the core in images A and B are offcenter and smaller than the core in images $\mathrm{C}$ and D. This changing air core position and size reflects that the air core has size and position fluctuations along the length of the PCGLS.

Figure 10 shows reconstructed gas core images for a constant water flow rate and increasing air flow rates. The imaged air cores are slightly off center, which is more noticeable at lower air flow rates (images $\mathrm{A}$ and $\mathrm{B}$ ). This becomes slightly less noticeable at higher air flow rates but the cores are still slightly off-center. This is reflected in Figure 5 where the cores are observed to be off-centric, more so at low air flow rates. These images can be analyzed to determine the gas core radius by measuring between the dark blue pixels. The measured gas core radii from the DCPT reconstructed images are summarized in Table 1.These reconstructed images can also be analyzed to determine the liquid holdup throughout the sensor. Table 2 compares the imaged liquid holdup to the expected liquid holdup based on Figure 6.

\section{PCGLS liquid holdup measurement}

The liquid holdup can also be measured without the reconstructed image by directly using the data from the ECVT sensor. The real time liquid holdup is calculated by using a predetermined equation that relates the DCPT measurements to the liquid holdup. The air void fraction $\eta_{\text {air }}$ can then easily be obtained from the liquid holdup $\eta_{l h}$ as:

$$
\eta_{\text {air }}=1-\eta_{l h}
$$

Table 3 shows the visually estimated liquid holdup compared with the DCPT determined liquid holdup at varying water and air flow rates. 
Sines et al. -- Study of Flow in a Phase Separator Using DCPT
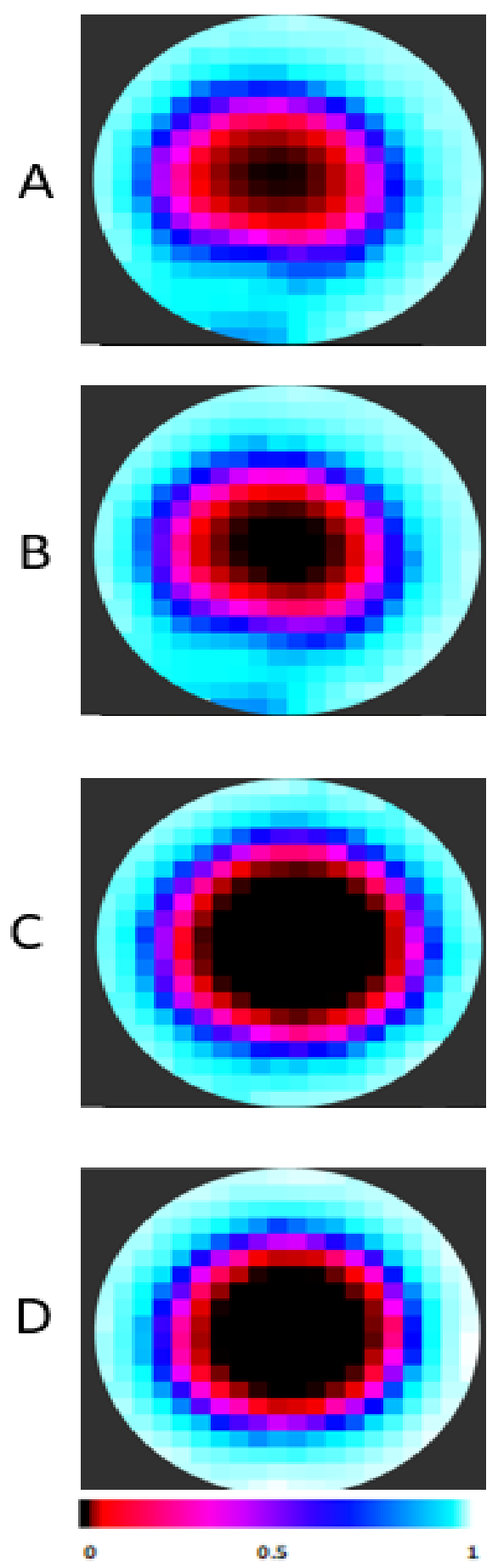

Figure 9. Typical displacement-current phase tomography cross section image reconstruction of liquid and gas distribution inside the passive cyclonic gas-liquid separators: (A) Top of Sensing Region, (B) 1/3 into Sensing Region, (C) 2/3 into Sensing Region, and (D) Bottom of Sensing Region. These images are from a 13.21 LPM, 12.27 SLPM test.
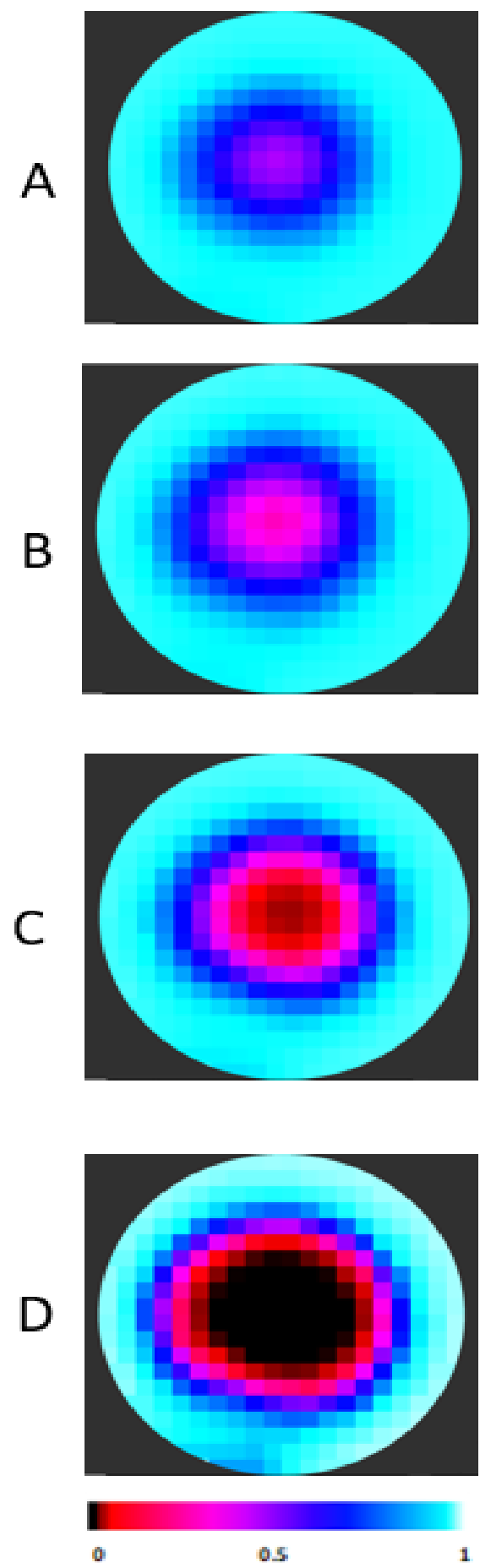

Figure 10. Displacement-current phase tomography reconstructed cross section images at a constant water injection rate $(13.27 \mathrm{~L} / \mathrm{min})$ and varying air injection rates: (A) 2.36 SLPM, (B) 5.19 SLPM, (C) 8.02 SLPM, and (D) 12.27 SLPM. 


\section{Sines et al. -- Study of Flow in a Phase Separator Using DCPT}

Table 1. Expected (from Figure 6) and imaged gas core radii at varying air flow rates. Liquid flow is held constant at 13.21 LPM for these results.

\begin{tabular}{|c|c|c|c|} 
Image & $\begin{array}{c}\text { Air Flow } \\
\text { Rate } \\
\text { (SLPM) }\end{array}$ & $\begin{array}{c}\text { Expected } \\
\text { Gas Core } \\
\text { Radius } \\
\mathbf{( m )}\end{array}$ & $\begin{array}{c}\text { Imaged } \\
\text { Gas Core } \\
\text { Radius } \\
\mathbf{( m )}\end{array}$ \\
\hline A & 2.36 & 0.0202 & 0.0187 \\
\hline B & 5.19 & 0.0232 & 0.0234 \\
\hline C & 8.02 & 0.0258 & 0.0251 \\
\hline D & 12.26 & 0.0283 & 0.0266 \\
\hline
\end{tabular}

Table 2. Expected and imaged average liquid holdup at varying air flow rates. Liquid flow is held constant at 13.21 LPM for these results.

\begin{tabular}{|c|c|c|c|} 
Image & $\begin{array}{c}\text { Air Flow } \\
\text { Rate } \\
\text { (SLPM) }\end{array}$ & $\begin{array}{c}\text { Expected } \\
\text { Liquid } \\
\text { Holdup }\end{array}$ & $\begin{array}{c}\text { Imaged } \\
\text { Liquid } \\
\text { Holdup }\end{array}$ \\
\hline A & 2.36 & 0.72 & 0.75 \\
\hline B & 5.19 & 0.63 & 0.62 \\
\hline C & 8.02 & 0.54 & 0.56 \\
\hline D & 12.26 & 0.45 & 0.51 \\
\hline
\end{tabular}

The measurements in Table 3 have an average absolute error of $5.80 \%$ between the visually determined and DCPT determined liquid holdups (error calculated as the absolute value of the difference between visual and DCPT holdup measurements). Some sources of this error may include not fully accounting for image distortion in the visual measurement and the potential that either peak in the periodic shape was measured rather than the gas core size.

As can be seen in Figure 11, the liquid holdup inside the separator tends to follow a periodic shape. This shape is to be expected due to the pulsating of the gas core as described in the $P C G L S$ section above. When the gas core pulses outwards, the liquid holdup decreases, and when the gas core pulses inwards, the liquid holdup increases, creating a periodic shape. This periodic fluctuation in the signal is much more noticeable at higher air flow rates, as was also discussed in the PCGLS section. It can also be seen that the liquid holdup signal is noisier at lower air flow rates. This is probably due to the smaller size of the gas core under these conditions. If the gas core is too small then liquid will exit from both the liquid outlet and the air outlet (Ellis, 2006). This action of liquid exiting through the air outlet makes the gas core less stable, causing the liquid holdup signal to appear noisier. As the gas core size increases, less water exits from the air outlet until the system becomes stable with only water exiting from the liquid outlet and only air exiting from the gas outlet. When the system reaches this stability point the gas core stabilizes, causing the liquid holdup signal to become less noisy.

Table 3. Visual and displacement-current phase tomography (DCPT) measured liquid holdup at varying air and water flow rates. Highlighted rows are the four cases imaged in Figure 10.

\begin{tabular}{|c|c|c|c|}
\hline $\begin{array}{c}\text { Water Flow } \\
\text { Rate } \\
\text { (LPM) }\end{array}$ & $\begin{array}{c}\text { Air Flow } \\
\text { Rate } \\
\text { (SLPM) }\end{array}$ & $\begin{array}{c}\text { Average } \\
\text { Liquid } \\
\text { Holdup } \\
\text { (Visual) }\end{array}$ & $\begin{array}{c}\text { Average } \\
\text { Liquid } \\
\text { Holdup } \\
\text { (DCPT) }\end{array}$ \\
\hline 14.213 & 2.36 & 0.899 & 0.871 \\
\hline 14.213 & 3.78 & 0.873 & 0.824 \\
\hline 14.213 & 5.19 & 0.87 & 0.825 \\
\hline 14.213 & 6.61 & 0.867 & 0.787 \\
\hline 14.213 & 8.02 & 0.7 & 0.648 \\
\hline 14.213 & 9.44 & 0.72 & 0.667 \\
\hline 14.213 & 10.85 & 0.68 & 0.510 \\
\hline 14.213 & 12.27 & 0.621 & 0.575 \\
\hline $\mathbf{1 3 . 2 6 5}$ & $\mathbf{2 . 3 6}$ & $\mathbf{0 . 7 2}$ & $\mathbf{0 . 6 5 1}$ \\
\hline $\mathbf{1 3 . 2 6 5}$ & 3.78 & 0.6 & 0.497 \\
\hline $\mathbf{1 3 . 2 6 5}$ & $\mathbf{5 . 1 9}$ & $\mathbf{0 . 6 3}$ & $\mathbf{0 . 5 4 7}$ \\
\hline $\mathbf{1 3 . 2 6 5}$ & 6.61 & 0.55 & 0.478 \\
\hline $\mathbf{1 3 . 2 6 5}$ & $\mathbf{8 . 0 2}$ & $\mathbf{0 . 5 4}$ & $\mathbf{0 . 5 7 1}$ \\
\hline $\mathbf{1 3 . 2 6 5}$ & 9.44 & 0.53 & 0.437 \\
\hline $\mathbf{1 3 . 2 6 5}$ & 10.85 & 0.42 & 0.343 \\
\hline $\mathbf{1 3 . 2 6 5}$ & $\mathbf{1 2 . 2 7}$ & $\mathbf{0 . 4 5}$ & $\mathbf{0 . 4 1 8}$ \\
\hline 12.318 & 2.36 & 0.58 & 0.505 \\
\hline 12.318 & 3.78 & 0.56 & 0.506 \\
\hline 12.318 & 5.19 & 0.487 & 0.4855 \\
\hline 12.318 & 6.61 & 0.464 & 0.473 \\
\hline 12.318 & 8.02 & 0.422 & 0.484 \\
\hline 12.318 & 9.44 & 0.403 & 0.500 \\
\hline 12.318 & 10.85 & 0.384 & 0.464 \\
\hline 12.318 & 12.27 & 0.352 & 0.461 \\
\hline
\end{tabular}




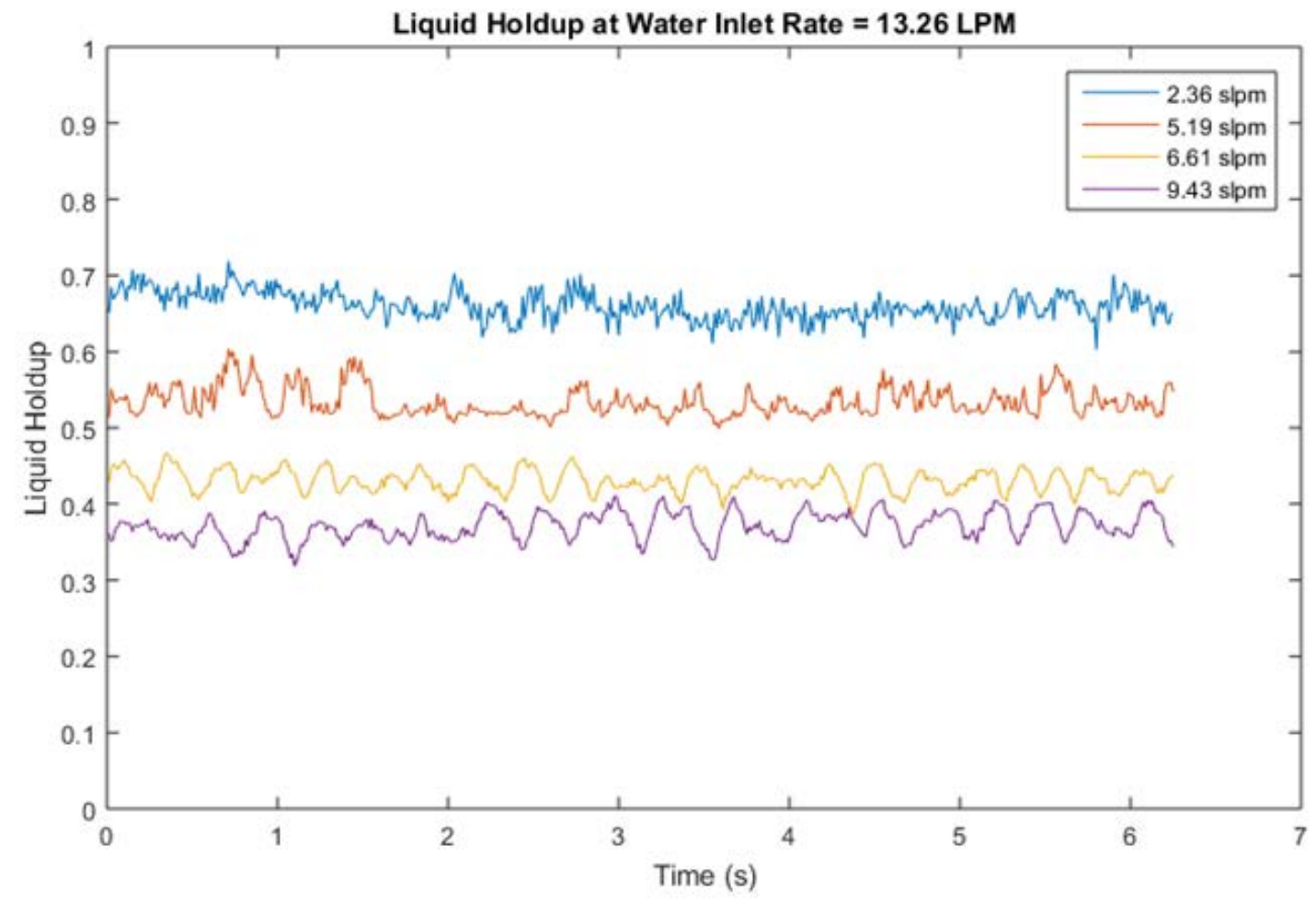

Figure 11. Liquid holdup over time for a water flow rate of 19 LPM and air flow rates varying from 4.75 SLPM to 9.4 SLPM. The signals from top to bottom correspond to air flow rates of: 4.75 SLPM, 5.6 SLPM, 6.6 SLPM, 7.5 SLPM, and 9.4 SLPM.

\section{PCGLS angular velocity}

The basic model for a PCGLS consists of a steady air core in the center of a rotating water layer. In reality, this air core is generally unsteady with pulsations and local flow structures in the liquid stream. DCPT can capture these irregularities in the air core by analyzing the signal variations from different capacitance pairs. The angular velocity of the local flow structure can thus be calculated through cross correlation of the signal variations. Plate pairs within the same layer with $60^{\circ}$ separation are chosen for this angular velocity analysis. The number of frames between local maxima in a single plate pair signal are determined and used in conjunction with the data capture rate to determine the angular velocity of the water layer inside the separator.

$$
\Omega(R P M)=\frac{\text { Data Capture Rate } * 60}{\text { frames }}
$$

Figure 12 shows the DCPT determined angular velocity values plotted against the tachometer determined values from Figure 7. It can be seen that both measurement methods, DCPT and Tachometer, give similar results for each air and water flow rate. It is also observed that at a constant water flow rate, the angular velocity tends to increase linearly with the air flow rate. A linear fit applied to the angular velocity data suggests that the relation between angular velocity and air flow rate, when air flow rate is greater than zero, is as follows:

$$
\Omega(r p m)=17.95 * Q(S L P M)+B-40
$$

where Q is the air flow rate in SLPM and B is the angular velocity under liquid-only injection conditions. Equation 2 can be substituted for B in Equation 5, giving the relation between air flow rate, water flow rate, and angular velocity for this study as:

$$
\begin{gathered}
\Omega(r p m)=-17.95 * Q(S L P M)+59.26 \\
* W(L P M)-223
\end{gathered}
$$



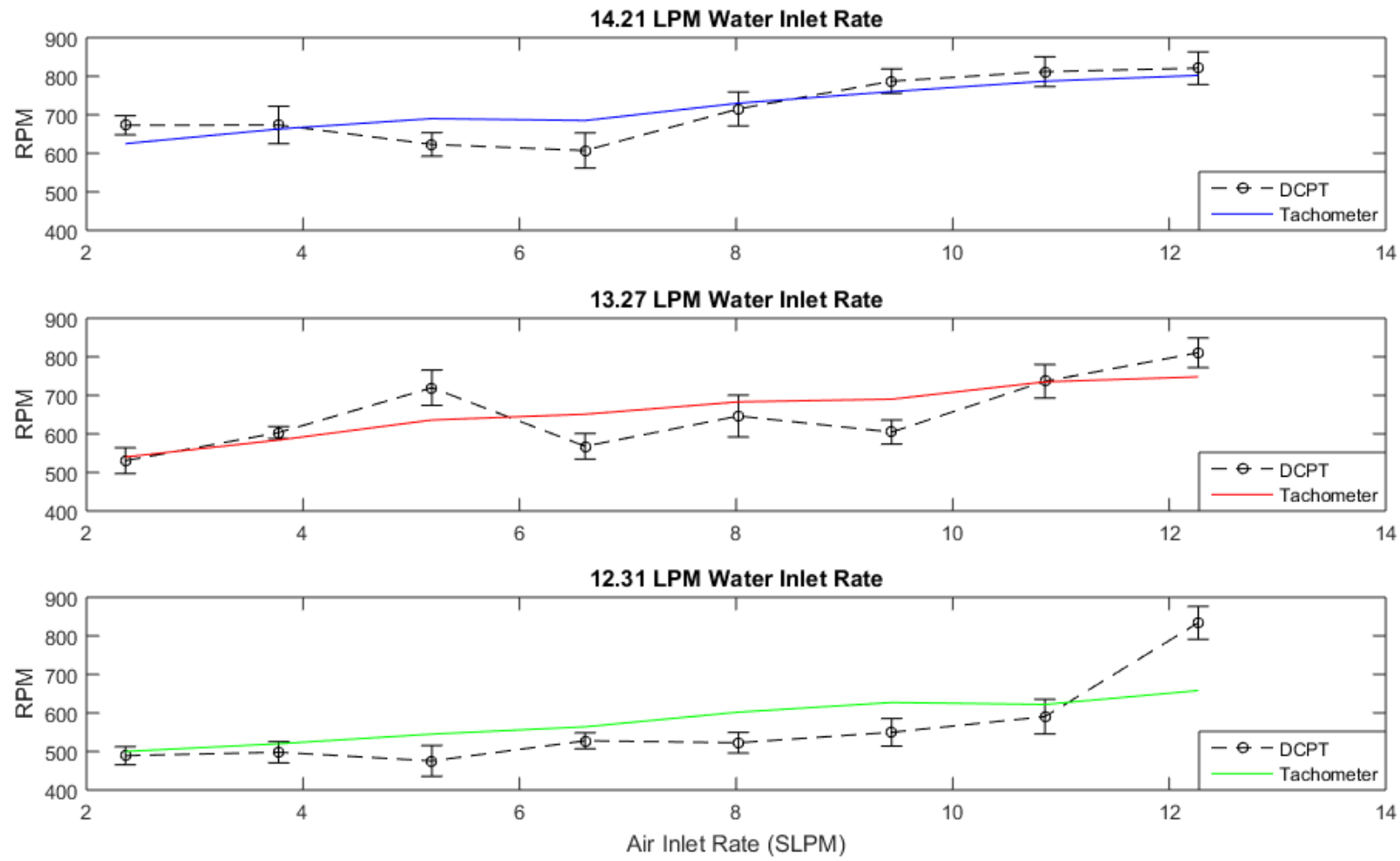

Figure 12. Displacement-current phase tomography determined angular velocities plotted against the tachometer determined angular velocity values. The vertical error bars represent the standard deviation in the DCPT angular velocity measurements.

A potential source of error in the DCPT measurements is noise in the data signal. The noise in the data signal can affect the system's ability to detect the air core irregularities that are used to calculate angular velocity. This noise will also affect instantaneous liquid holdup readings but can be mostly averaged out if data is collected for a long enough time.

\section{Air-water inlet flow liquid holdup}

Nineteen different combinations of air and water flow rates are tested. Seven of these conditions are tested in the up flow section and twelve are tested in the down flow section. The up flow combinations are tested three times and the down flow combinations are tested twice. The down flow combinations are only tested twice due to time constraints on access to the test flow loop.
Once again the real time liquid holdup is calculated using a predetermined equation relating the ECVT measurements to the liquid holdup.

Table 4 compares the visual liquid holdup measurements, measured with the QCVs, with the ECVT liquid holdup measurements for the nineteen flow combinations. For simplicity the three up flow tests are averaged together and the two down flow tests are averaged together to get a single value for each flow combination in each flow direction. The absolute error and relative error between the visual and ECVT measurements are plotted together in Figure 13. The absolute error is calculated as the absolute value of the difference between the visual and ECVT measurements. The relative error is calculated using equation 7 below:

$$
\text { Relative Error }(\%)=\frac{\mid \text { Visual }-E C V T \mid}{\text { Visual }} * 100
$$


Sines et al. -- Study of Flow in a Phase Separator Using DCPT

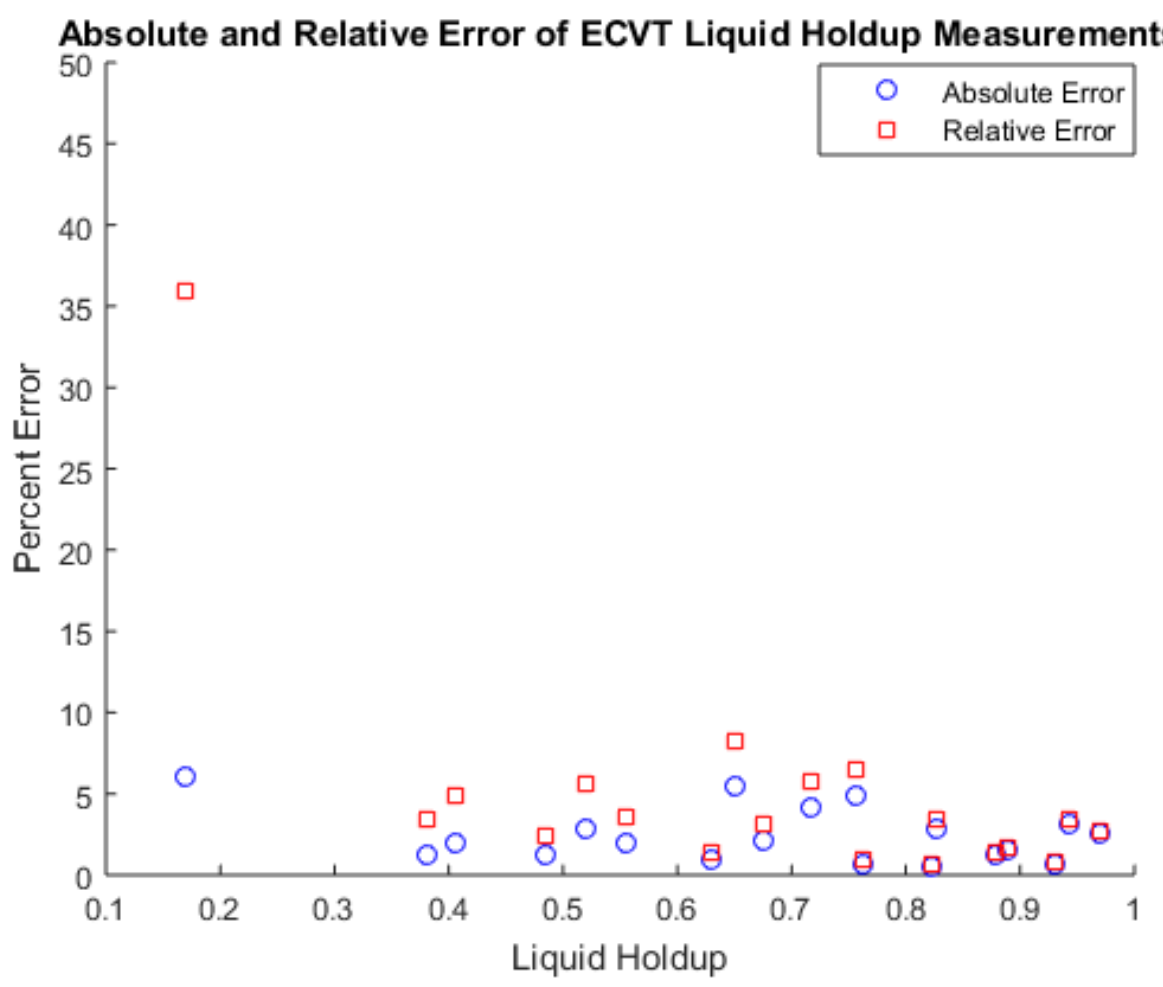

Figure 13. Absolute and relative error between the visual and electrical capacitance phase tomography liquid holdup measurements within the air-water inlet flow test.

Table 4. Visual and electrical capacitance phase tomography (ECVT) measurements of air-water vertical flow.

\begin{tabular}{|c|c|c|c|c|}
\hline $\begin{array}{c}\text { Flow } \\
\text { Direction }\end{array}$ & $\begin{array}{c}\text { Water Flow } \\
\text { Rate (LPM) }\end{array}$ & $\begin{array}{c}\text { Air Flow Rate } \\
\text { (SLPM) }\end{array}$ & $\begin{array}{c}\text { Measured Liquid } \\
\text { Holdup (Visual) }\end{array}$ & $\begin{array}{c}\text { Measured Liquid } \\
\text { Holdup (ECVT) }\end{array}$ \\
\hline Up & 18.95 & 3 & 0.969 & 0.943 \\
\hline Up & 18.95 & 5 & 0.930 & 0.923 \\
\hline Up & 18.95 & 8 & 0.879 & 0.891 \\
\hline Up & 18.95 & 12 & 0.827 & 0.855 \\
\hline Up & 18.95 & 18 & 0.757 & 0.806 \\
\hline Up & 18.95 & 24 & 0.717 & 0.758 \\
\hline Up & 18.95 & 34 & 0.650 & 0.704 \\
\hline Down & 18.95 & 3 & 0.943 & 0.911 \\
\hline Down & 18.95 & 5 & 0.890 & 0.875 \\
\hline Down & 18.95 & 8 & 0.822 & 0.827 \\
\hline Down & 18.95 & 12 & 0.762 & 0.769 \\
\hline Down & 18.95 & 18 & 0.675 & 0.696 \\
\hline Down & 18.95 & 24 & 0.630 & 0.639 \\
\hline Down & 18.95 & 34 & 0.555 & 0.575 \\
\hline Down & 18.95 & 40 & 0.520 & 0.549 \\
\hline Down & 18.95 & 50 & 0.485 & 0.497 \\
\hline Down & 15.16 & 50 & 0.405 & 0.425 \\
\hline Down & 13.25 & 50 & 0.380 & 0.367 \\
\hline Down & 3.79 & 30 & 0.170 & 0.109 \\
\hline
\end{tabular}




\section{Sines et al. -- Study of Flow in a Phase Separator Using DCPT}

It can be seen that the ECVT measurements and visual measurements closely match with an average absolute error of $2.44 \%$ and an average relative error of $5.03 \%$.

$90 \%$ of the ECVT measurements are below $5 \%$ absolute error and 95\% are below 10\% relative error. There is some inherent error between the ECVT and QCV measurements as they are not necessarily measuring the same liquid holdup. The QCV measurements give an average liquid holdup by assuming that the liquid holdup is constant throughout the test region. In reality the liquid holdup varies across the test region and is constantly fluctuating as the water and air flow through. The ECVT system measures these fluctuations in real time meaning that the average ECVT measurement is an average of the liquid holdup fluctuations. This is why the absolute error is greater in tests where liquid holdup fluctuations were larger, specifically the high air flow tests within the up flow section.

\section{DISCUSSION}

In this study, the angular velocity and liquid holdup inside of a horizontal passive cyclonic gasliquid separator is investigated using DCPT, an extension of ECVT. The liquid holdup inside the air-water injection flow is also investigated. The PCGLS gas core diameter, angular velocity of the liquid layer and liquid holdup inside the separator and two phase injection flow are measured both visually and using DCPT. The two methods are then compared to each other to determine accuracy of the DCPT measurements. It is found that the gas core diameter increases in a logarithmic shape as the gas flow is increased. It is also observed that the gas core pulsates in and out, and that this motion is more noticeable at higher gas flow rates. Finally, it is found that the angular velocity increases linearly with water flow rate when only liquid is injected. The angular velocity also increases linearly with air flow rate when the water flow rate is kept constant. A model is then derived to determine the angular velocity of the liquid velocity given the water and air flow rates.

\section{ACKNOWLEDGEMENTS}

This work was supported by the National Aeronautics and Space Administration (NASA)
Phase II Small Business Innovation Research (SBIR) project under grant NNX16CC10C. The project was in support of Space Life \& Physical Sciences Division funding for the Phase Separator.

\section{REFERENCES}

Barbu A, Ellis M, Kurwitz C, Best F (2006) Acoustic gauge monitoring of fluid inventory in a microgravity vortex separator. Measurement and Science Technology 17(2): 403-410

Ellis M (2006) The Tangential Velocity Profile and Momentum Transfer Within a Microgravity Vortex Separator (Master Dissertation), Texas A\&M University

Gunes C, Marashdeh Q, Teixeira FL (2017) A comparison between electrical capacitance tomography and displacement-current phase tomography. IEEE Sensors Journal 17(24): 8037-8046

Hoyt N (2013) The Performance of Passive Cyclonic Separators in Microgravity (Doctorate Dissertation), Case Western Reserve University

Hoyt NC, Kang MF, Kharraz A, Kadambi J, Kamotani Y (2011) Cyclonic two-phase flow separator experimentation and simulation for use in a microgravity environment. Journal of Physics: Conference Series 327: 012-056

Hoyt NC, Kang MF, Lee KL, Kharraz A, Kadambi J, Kamotani Y (2013) Study of steady and dynamic behavior of gas core of passive cyclonic separator for space applications. Microgravity Science and Technology 25(3): 187-200

Kang MF, Hoyt NC, Kadambi J, Kamotani Y (2014) Study of gas core behavior of passive cyclonic two-phase separator for microgravity applications. Microgravity Science and Technology 26(3): 147-157

Lowe DC, Rezkallah KS (1999) Flow regime identification in microgravity two-phase flows using void fraction signals. International Journal of Multiphase Flow 25: 433-457

Wang A, Marashdeh Q, Fan LS (2016) ECVT imaging and model analysis of the liquid distribution inside a horizontally installed 


\section{Sines et al. -- Study of Flow in a Phase Separator Using DCPT}

passive cyclonic gas-liquid separator. Chemical Engineering Science 141: 231-239

Wang A, Marashdeh Q, Teixeira FL, Fan LS (2015) Electrical capacitance volume tomography: a comparison between 12- and 24-channels sensor systems. Progress in Electromagnetics Research M 41: 73-84

Warsito W, Marashdeh Q, Fan LS (2007) Electrical capacitance volume tomography. IEEE Sensors Journal 7(3-4): 525-535 